\title{
DÜBLIN
}

Technological University Dublin

ARROW@TU Dublin

\section{On the distortion of UWB circularly polarized time-domain pulses in presence of rotation}

\author{
Adam Narbudowicz \\ Wroclaw University of Science and Technology \\ Janusz Przewocki \\ University of Gdansk \\ Max Ammann \\ Technological University Dublin, max.ammann@tudublin.ie
}

Follow this and additional works at: https://arrow.tudublin.ie/ahfrccon

Part of the Other Electrical and Computer Engineering Commons, and the Systems and Communications Commons

\section{Recommended Citation}

A. Narbudowicz, J. Przewocki and M. J. Ammann, "On the Distortion of UWB Circularly Polarized TimeDomain Pulses in Presence of Rotation," 2019 IEEE International Symposium on Antennas and Propagation and USNC-URSI Radio Science Meeting, 2019, pp. 1735-1736, doi: 10.1109/ APUSNCURSINRSM.2019.8888922.

This Conference Paper is brought to you for free and open access by the Antenna \& High Frequency Research Centre at ARROW@TU Dublin. It has been accepted for inclusion in Conference Papers by an authorized administrator of ARROW@TU Dublin. For more information, please contact arrow.admin@tudublin.ie, aisling.coyne@tudublin.ie,gerard.connolly@tudublin.ie.

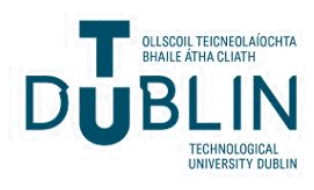




\title{
On the Distortion of UWB Circularly Polarized Time-Domain Pulses in Presence of Rotation
}

\author{
Adam Narbudowicz \\ Department of Telecommunications and Teleinformatics \\ Wroclaw University of Science and Technology \\ Wroclaw, Poland \\ adam.narbudowicz@pwr.edu.pl
}

\author{
Janusz Przewocki \\ Institute of Mathematics \\ University of Gdansk \\ Gdansk, Poland \\ jprzew@mat.ug.edu.pl
}

\author{
Max J. Ammann \\ Antenna \& High Frequency Research Centre \\ TU Dublin \\ Dublin, Ireland \\ max.ammann@dit.ie
}

\begin{abstract}
The paper provides a first theoretical study on the effect of rotational Doppler on circularly polarized pulsed communication. Despite the circularly polarized communication being considered immune to signal fading due to rotary misalignment, such misalignment will cause a frequency-invariant phase-shift. This phase shift will significantly distort the shape of the time-domain pulse. The property can be used for integration of orientation sensing into well establish pulse-based localization. However, it has also the potential to distort communication for some pulse-modulated UWB systems.
\end{abstract}

Keywords-Ultra-Wideband; UWB; Pulse modulation; Circular Polarization; Localization; Wireless sensing

\section{INTRODUCTION}

Circularly Polarized (CP) antennas have been long used for communication between rotating objects (e.g. satellite communication, RFID) as they are immune to polarization losses due to misalignment [1]. In the presence of a rotation between transmitter and receiver antennas, the amplitude of the $\mathrm{CP}$ signal is not affected; however its phase will change proportionally to the rotation angle - a phenomena known as the rotational Doppler Effect [2]. A recent study demonstrated that the phenomena can be used to accurately measure rotational speeds of various devices, with very high dynamic range [3]. For instance: a rotating speed of 600M RPM (which was reported in [4] as the fastest man-made rotating object) would produce a frequency shift of $10 \mathrm{MHz}$, which falls well within measurement capabilities of state-of-the-art RF measurement equipment.

While the single-frequency signal allows measurement of rotating objects at a fixed-distance, it fails for more dynamic cases since the system cannot distinguish whether the phase shift originated from rotational or linear movement. However,

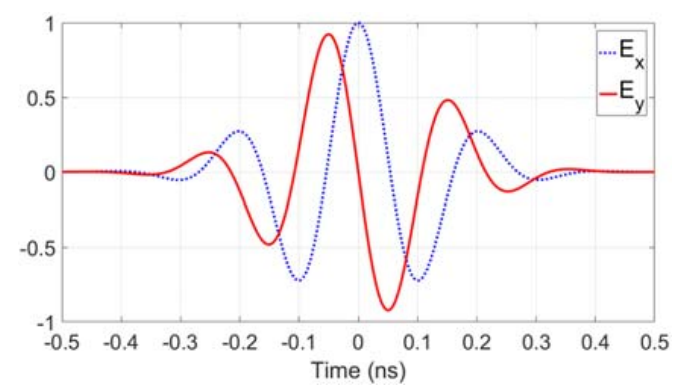

a)

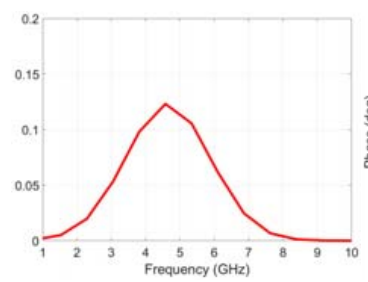

b)

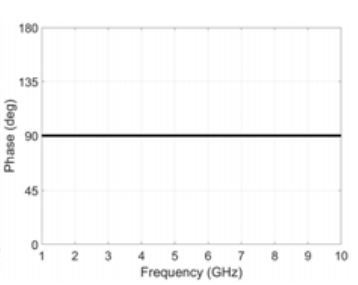

c)
Fig. 1: $E_{x}$ and $E_{y}$ components of the CP Gaussian pulse: a) time-domain; b) amplitude of the spectrum; c) phase difference between $E_{x}$ and $E_{y}$ spectrum

contrary to the translational Doppler effect, the rotational case is frequency invariant, i.e. rotation by an angle $\alpha$ will produce a frequency shift of $\alpha$ at all frequencies (assuming the conditions for CP are satisfied) [5]. This property was used for dualfrequency implementation that allows simultaneous measurement of rotation and distance change [6]. However due to the lack of redundancy the technique was prone to errors. 
This paper investigates for the first time the use of UltraWide Band (UWB) time-domain CP pulses [7] for measurement of the rotation. It provides a first theoretical study on how rotation influences the shape of UWB pulses and - most importantly - that such influence is significantly different from the one experienced by distance change. Since UWB pulses have been extensively used for accurate localization with $<20$ $\mathrm{cm}$ resolution [8], the implementation of rotation sensing based on the same technology is very attractive, due to easy integration capabilities.

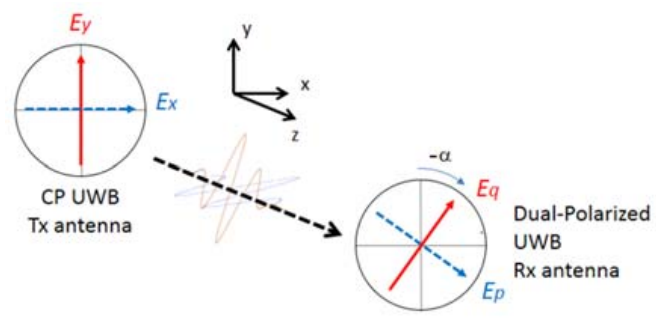

Fig. 2: Investigated scenario

\section{MATHEMATICAL MODEL}

Fig. 1 demonstrates the two orthogonal components of a CP Gaussian pulse, representing frequencies from 3.2 to $6.2 \mathrm{GHz}$. This corresponds to the $3 \mathrm{~dB}$ Axial Ratio (AR) band of the antenna proposed in [9]. It was ensured that the spectrum of $E_{x}$ and $E_{y}$ components have the same amplitudes and are shifted in phase by $90^{\circ}$, thus providing an $\mathrm{AR}=0$ at all investigated frequencies.

Fig. 2 depicts the investigated scenario. The transmit antenna is an UWB CP antenna, transmitting the pulse with component $E_{x}$ and $E_{y}$. The receive antenna is dual linearlypolarized, measuring independently two orthogonal components $E_{p}$ and $E_{q}$, which lie in the plane orthogonal to the propagation direction of the pulse. The measured components $E_{p}$ and $E_{q}$ are rotated with respect to $E_{x}$ and $E_{y}$ by angle $\alpha$. This can be described as:

$$
\left[\begin{array}{l}
E_{p} \\
E_{q}
\end{array}\right]=\left[\begin{array}{cc}
\cos \alpha & -\sin \alpha \\
\sin \alpha & \cos \alpha
\end{array}\right]\left[\begin{array}{l}
E_{x} \\
E_{y}
\end{array}\right]
$$

\section{Pulse Distortion}

Based on (1) the shape of the pulse was calculated for various rotations $\alpha$ in negative direction from $-60^{\circ}$ to $-360^{\circ}$ in $60^{\circ}$ steps. Fig. 3a shows the shape of the pulses received by the detector measuring the $E_{p}$ component, while Fig $3 \mathrm{~b}-E_{q}$ component. It can be seen, that the rotational Doppler Effect causes modulation which significantly changes pulse's shape. This distortion is unique to rotary movement and is significantly different from time-delay caused by distance travelled (used in localization applications) and from shortened/prolonged pulse due to the translational Doppler Effect. While facilitating simultaneous measurement of orientation and localization

This project has received funding from the European Union's Horizon 2020 program under the Marie Skłodowska-Curie Actions agreement No 713567 and from Science Foundation Ireland (SFI) grant No. 13/RC/2077.
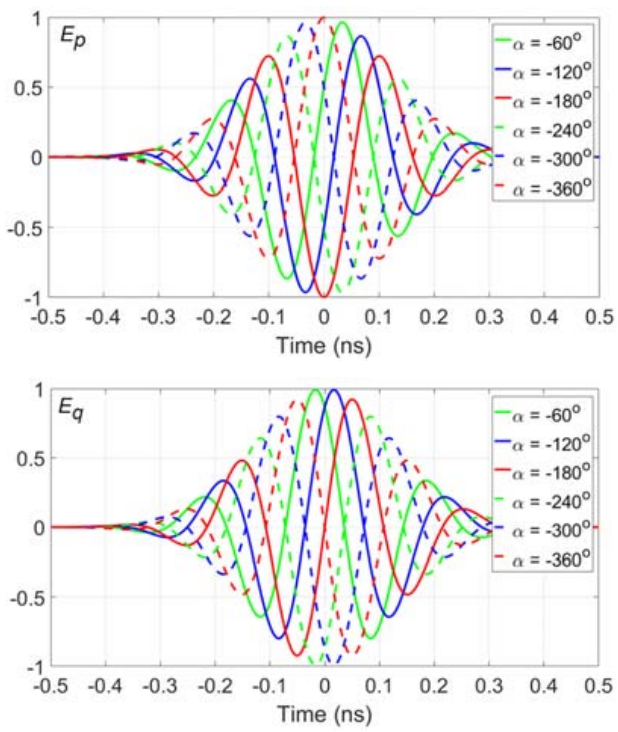

Fig. 3: $E_{p}$ and $E_{q}$ components of received pulses as a function of $\alpha$

integrated into a single UWB tag, the demonstrated pulse distortion may also impede communication when certain pulsemodulation techniques are used on rotating platforms (e.g. pulsed satellite communication).

\section{CONCLUSIONS}

The paper demonstrates theoretically the impact of rotary movement on UWB CP pulse. It shows a significant distortion of such pulse, which is caused by a frequency-invariant phase shift due to rotational Doppler Effect.

\section{REFERENCES}

[1] S. Gao, Qi Luo, Fuguo Zhu, "Circularly polarized antennas", WileyIEEE Press, January 2014

[2] G. Li, T. Zentgraf, S. Zhang, "Rotational Doppler effect in nonlinear optics", Nature Physics, vol. 12, pp. 736 - 740, 2016

[3] V. Sipal, A. Narbudowicz, M. J. Ammann, "Contactless Measurement of Angular Velocity Using Circularly Polarized Antennas" IEEE Sensors Journal, vol. 15, no 6, pp. 3459-3466, 2015

[4] Y. Arita, M. Mazilu, K. Dholakia, "Laser-induced rotation and cooling of a trapped microgyroscope in vacuum", Nature Communications, vol. 4, art. No. 2374, 2013

[5] V. Sipal, A. Narbudowicz, M. J. Ammann, "Using near-field coupled circularly polarised antennas as frequency-independent variable phase shifters", Electronics Letters, vol. 50, no. 11, pp. 788-790, 2014.

[6] A. Narbudowicz, M. J. Ammann, and D. Heberling, "Simultaneous rotation and distance measurement using multiband circularly polarized radio link", In Proc.: Int. Symp. on Ant. and Prop. (ISAP 2016), Okinawa, Japan, 24-28 Oct. 2016.

[7] A. Shlivinski, "Time-Domain Circularly Polarized Antennas", IEEE Trans. on Ant. and Prop., vol. 57, no 6, pp. 1606-1611, 2009.

[8] A. R. Jiménez Ruiz, F. Seco Granja, "Comparing Ubisense, BeSpoon, and DecaWave UWB Location Systems: Indoor Performance Analysis" IEEE Trans. on Inst. and Meas., vol. 66, no 8, pp. 2106-2117, 2017

[9] A. Narbudowicz, M. John, V. Sipal, X. Bao, and M. J. Ammann, "Design Method for Wideband Circularly Polarized Slot Antennas" IEEE Trans. on Ant. and Prop., vol. 63, no 10, pp. 4271-4279, 2015. 